\title{
A Stokes-based spectro-polarimetric analysis of the amplified spontaneous emission in a semiconductor optical amplifier
}

\author{
Mohamad Tariaki \\ Frédéric Boulvert \\ Florian F.L. Bentivegna \\ fb@enib.fr \\ Mikael Guégan
}

Joseph Topomondzo

\section{Ammar Sharaiha}

\section{Fabrice Pellen}

\section{Bernard Le Jeune}

\author{
RESO, EA 3380, ENIB, CS 73862, 29238 Brest, France \\ LSOL, EA 938, Université de Bretagne Occidentale, CS 93837, 29238 Brest, France
}

RESO, EA 3380, ENIB, CS 73862, 29238 Brest, France

RESO, EA 3380, ENIB, CS 73862, 29238 Brest, France

RESO, EA 3380, ENIB, CS 73862, 29238 Brest, France

RESO, EA 3380, ENIB, CS 73862, 29238 Brest, France

LSOL, EA 938, Université de Bretagne Occidentale, CS 93837, 29238 Brest, France

LSOL, EA 938, Université de Bretagne Occidentale, CS 93837, 29238 Brest, France

In this paper, we present a full spectro-polarimetric characterization of the Amplified Spontaneous Emission (ASE) emitted from a commercial, strained-bulk Semiconductor Optical Amplifier (SOA). A partly polarised, broadband noise source that draws from the same carrier reservoir as the amplified signal, ASE affects the state of polarisation of the overall outgoing signal of a SOA and its polarimetric behaviour is thus of interest. This study was carried out within the frame of the Stokes formalism, which not only allows a determination of the degree of polarisation (DOP) of ASE directly from its Stokes vector, but also gives access to a full, spectrally resolved characterization of its polarised fraction with respect to the applied bias current. Even when that fraction is small, its state of polarisation was shown to be strongly governed by the spectral dependence of the material gain upon polarisation. The same study was performed with a fully polarised signal injected into the SOA. [DOI: 10.2971/je0s.2007.07025]

Keywords: semiconductor optical amplifiers, amplified spontaneous emission, polarisation

\section{INTRODUCTION}

Mainly due to their multifunctional qualities [1, 2], Semiconductor Optical Amplifiers (SOAs) remain key devices for future all-optical communication systems, especially when optical signal processing is involved [3]. However, the fact that SOAs are inherently polarisation-dependent devices is an important disadvantage for most of these applications, for which polarisation insensitivity is a fundamental requirement. In standard systems, the optical signal necessitating amplification is most of the time injected into the SOA through a fibre and its polarisation state is thus usually random. For that reason, any polarisation dependence of the amplification efficiency is a limiting factor for the use of SOAs. Moreover the polarisation sensitivity of the optical gain and - as is too often forgotten - of the optical index of SOAs can be a major drawback for monolithic integration. Those are the reasons why numerous studies have been carried out in order to design and produce polarisation-insensitive structures i.e., such that the gain variation with the polarisation state of the incoming light does not exceed $0.5 \mathrm{~dB}$. In so doing, many approaches have been proposed for the geometry of the guide or for the gain medium of the active layer, and have led, for instance, to bulk structures with a nearly square cross-section [4], strained multi-quantum-well structures [5], and, more recently, strained-bulk rectangular structures [6, 7]. On the other hand, the possibility to exploit the polarisation sensitivity of SOAs has been widely investigated these last few years with the aim to optimise some optical functions [8] or to develop entirely new ones $[9,10]$.

In any case, a detailed polarimetric characterisation is required if a complete understanding of the polarisation dependence of the amplification process in a SOA is to be achieved. We previously reported on a full polarimetric study of a SOA within the frame of the Mueller-Jones formalism [11], which is a systematic polarimetric analysis much more suitable, in our view, than the Jones formalism previously reported [12], in presence of a partly polarised broadband source such as the Amplified Spontaneous Emission (ASE). Even within this frame, however, our previous work showed that ASE remains an issue [11], i.e., is a source of ill-defined polarisation noise, unless it is filtered out - as it draws from the same carrier reservoir as the amplified signal itself. Because the Mueller- 
Jones formalism is expected to bring valuable information on the origin of polarisation-dependent phenomena in SOAs, it is clearly desirable to seek an extension of this formalism to active systems presenting a nonlinear, broadband, partly polarised source such as ASE. However, a precise characterisation of the state of polarisation of ASE alone in a SOA is a necessary preamble to this project.

In this paper, we present a spectro-polarimetric investigation of ASE emitted from a single-mode, tensile-strained SOA. This device was thus designed in order to achieve a low polarisation dependency of its amplification factor. Section 2 introduces the Stokes formalism used to characterise the state of polarisation of ASE and to determine the parameters that define it - its degree of polarisation (DOP) as well as the azimuth and ellipticity of the characteristic ellipse of its polarised fraction. In Section 3 we present the experimental setup and procedure used for the determination of those polarimetric parameters. Section 4 details and discusses the experimental results, i.e., the behaviour of the polarimetric parameters as functions of the wavelength $\lambda$ and of the bias current $I_{\text {bias }}$ applied to the structure. The dependency of the state of polarisation of ASE upon that of an injected signal is also discussed.

\section{THE STOKES FORMALISM AND THE STATE OF POLARISATION OF ASE}

The Stokes formalism is a way to characterise the polarimetric features of any electromagnetic wave. Unlike the Jones formalism, it involves only real values, namely optical powers that can be experimentally determined. In the basis formed by the linear eigenmodes TE and TM of the single-mode structure, the components of the four-component Stokes vector $\mathbf{s}$ are defined as follows:

$$
\begin{gathered}
s_{0}=P_{T E}+P_{T M}=P_{t}, \\
s_{1}=P_{T E}-P_{T M}, \\
s_{2}=2 \sqrt{P_{T E} P_{T M}}<\cos (\Delta \phi)>, \\
s_{3}=2 \sqrt{P_{T E} P_{T M}}<\sin (\Delta \phi)>,
\end{gathered}
$$

where $P_{T E}$ and $P_{T M}$ are the mean optical powers associated to the TE and TM components of the optical electric field, $P_{t}$ is the total optical power, $\Delta \phi=\phi_{T M}-\phi_{T E}$ is the phase difference between these components, and the brackets $\langle\ldots\rangle$ denote a temporal averaging over the integration time of any detector used for the measurements.

In the most general case, an electromagnetic wave consists of the incoherent superposition of a fully polarised wave and of a fully non-polarised (or natural) wave. Hence its Stokes vector can be decomposed as follows:

$$
\mathbf{s}=\mathbf{s}_{\mathbf{p}}+\mathbf{s}_{\mathbf{u}}=\left(\begin{array}{c}
\sqrt{s_{1}^{2}+s_{2}^{2}+s_{3}^{2}} \\
s_{1} \\
s_{2} \\
s_{3}
\end{array}\right)+\left(\begin{array}{c}
s_{0}-\sqrt{s_{1}^{2}+s_{2}^{2}+s_{3}^{2}} \\
0 \\
0 \\
0
\end{array}\right)
$$

where $\mathbf{s}_{\mathbf{p}}$ and $\mathbf{s}_{\mathbf{u}}$ are the Stokes vectors of the polarised and unpolarised fractions of the optical wave, respectively.

One of the main interests of the Stokes formalism is that it allows a determination of the degree of polarisation (DOP) of any optical signal directly from its Stokes components. The $D O P$ quantifies that fraction of the total optical signal that is actually polarised, with:

$$
D O P=\frac{\sqrt{s_{1}^{2}+s_{2}^{2}+s_{3}^{2}}}{s_{0}}, \quad 0 \leq D O P \leq 1 .
$$

In the case of ASE, and in agreement with the very definition of the degree of polarisation, the DOP of its fully polarised fraction is obviously equal to 1 , whereas the DOP of its unpolarised fraction is zero.

As mentioned above, the components of the Stokes vector also lead to the determination of the characteristic ellipse of the polarised fraction of ASE emitted from the SOA (Figure 1). This

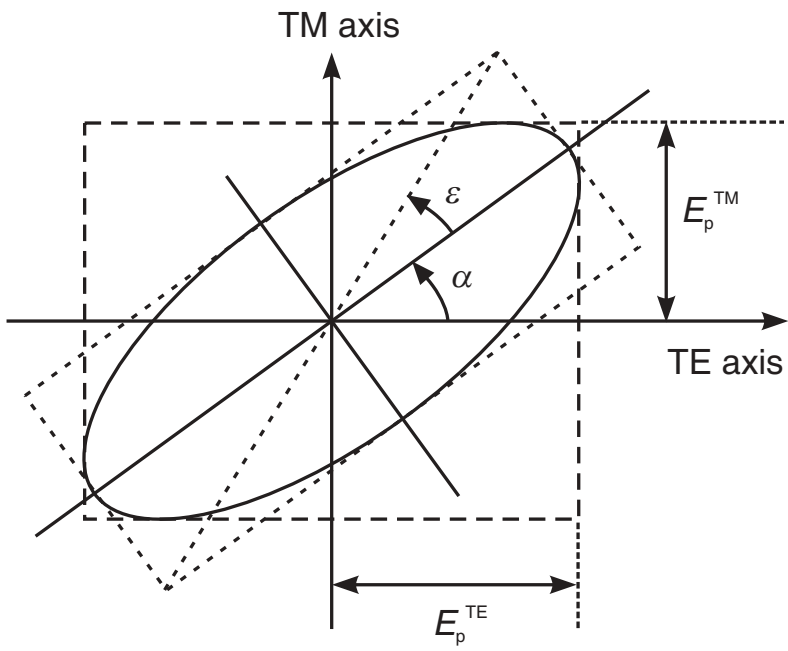

FIG. 1 Parameters of the characteristic ellipse of a polarised optical wave ( $\alpha$ : polarisa tion azimuth or orientation; $\epsilon$ : ellipticity; $E_{p}^{T E}$ and $E_{p}^{T M}$ : amplitudes of the TE and TM components of the optical electric field).

ellipse is determined by the temporal evolution, in any given waveplane, of the components $E_{p}^{T E}$ and $E_{p}^{T M}$ of the electric field associated to that fraction of ASE. Three parameters the polarisation azimuth (or orientation) $\alpha$ of the ellipse with respect to the TE axis of the structure, the ellipticity angle $\epsilon$, and the phase difference $\Delta \phi$ between the TE and TM components of ASE - entirely define the shape of this ellipse as well as its helicity, and they can indeed be shown to depend upon the components of the total Stokes vector, with:

$$
\tan (2 \alpha)=\frac{s_{2}}{s_{1}}, \quad 0 \leq \alpha<\pi,
$$

$$
\begin{gathered}
\sin (2 \epsilon)=\frac{s_{3}}{\sqrt{s_{1}^{2}+s_{2}^{2}+s_{3}^{2}}}, \quad-\pi / 4 \leq \epsilon \leq \pi / 4, \\
\tan (\Delta \phi)=\frac{s_{3}}{s_{2}}, \quad-\pi \leq \Delta \phi<\pi .
\end{gathered}
$$


The Stokes vector of ASE can then be entirely redefined as a function of the total ASE power $s_{0}$ emitted from the structure and of the geometrical parameters of its polarisation ellipse :

$$
\mathbf{s}=\mathbf{s}_{\mathbf{p}}+\mathbf{s}_{\mathbf{u}}=s_{0}\left(\begin{array}{c}
1 \\
\cos (2 \epsilon) \cos (2 \alpha) \\
\cos (2 \epsilon) \sin (2 \alpha) \\
\pm \sin (2 \epsilon)
\end{array}\right)
$$

where the sign of the fourth component is determined by the helicity of the ellipse - positive (respectively, negative) for a right-handed (respectively, left-handed) ellipse.

\section{EXPERIMENTAL SET-UP AND MEASUREMENT PROCEDURE}

This study was carried out on a bulky, tensile-strained, $500 \mu \mathrm{m}$ long InP/GaInAsP commercial semiconductor optical amplifier. The bulk active layer is $2 \mu \mathrm{m}$ wide and $0.2 \mu \mathrm{m}$ thick. Light propagates along the largest dimension of the structure, and the entrance and output facets of the guiding structure are both anti-reflection-coated for wavelengths centred around $1550 \mathrm{~nm}$, as well as slightly tilted with respect with the guide axis in order to further reduce their residual reflectivity. The geometry of such an ideally rectangular, single-mode structure favours two linearly-polarised, orthogonal propagation eigenmodes, TE and TM. The modal gain dependence of this SOA does not exceed $2 \mathrm{~dB}$ for a total gain of approximately 20 $\mathrm{dB}$ at a maximum value of the bias current $I_{\text {bias }}=225 \mathrm{~mA}$.

The polarimetric measurements were carried out in free space, which allows optimum control and preservation of the polarisation state of the collected signal.

Figure 2 schematically shows the experimental set-up that was used for the full, Stokes-based determination of the polarisation state of ASE emitted from the structure. The SOA

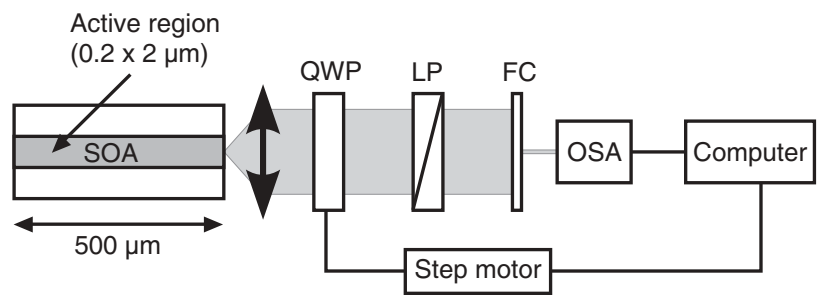

FIG. 2 Experimental set-up for the determination of the Stokes parameter of an optical wave (SOA: semiconductor optical amplifier; QWP: quarter wave plate; LP: linear polariser; FC: fibred collimator; OSA: optical spectrum analyser).

was positioned so that its TM and TE axes correspond to the vertical and horizontal axes of the laboratory referential, respectively. Light emitted from the SOA was collected and collimated with a microscope objective, then passed through a quarter-wave plate and a linear polariser acting as an analyser, before being recollected with a fibred collimator (diameter: $1 \mathrm{~cm}$ ) connected to an optical spectrum analyser (OSA). The passing axis of the linear polariser, when set vertically, coincided with the TM axis in the sample and defined a reference direction from which the orientation $\theta$ of the fast axis of the quarter-wave plate was estimated. This orientation could be modified, as the quarter-wave plate was mounted on a rotation stage whose movements were precisely determined by a computer-controlled step motor. The resolution of the OSA was $0.07 \mathrm{~nm}$.

For our study, the precise determination of all four components of the Stokes vector of ASE is essential. It can easily be shown that for any orientation $\theta$ of the linear analyser in the experimental set-up described above, the optical power $P(\theta)$ reaching the detector writes:

$$
P(\theta)=\frac{1}{2}\left[s_{0}+\cos ^{2}(2 \theta) s_{1}+\cos (2 \theta) \sin (2 \theta) s_{2}-\sin (2 \theta) s_{3}\right] .
$$

As a result, the complete determination of the four components of a Stokes vector requires four independent values of $\theta$, i.e., four different values of the measured optical power. In this study, though, for each experimental configuration (i.e., for each value of the wavelength $\lambda$ and of the bias current $\left.I_{\text {bias }}\right)$ the optical power $P(\theta)$ was measured for 24 orientations of the quarter-wave plate regularly spaced between 0 and $\pi$ radians, which means that each of the components of the Stokes vector was deduced from the average of 6 independent measurements. The aim of such an over-determination is to reduce the influence of random errors due to fluctuations of the state of polarisation of ASE.

For the determination of the ASE emission spectra, the quarter-wave plate was removed and the TE (when the analyser's passing axis is horizontal), TM (when it is vertical) and total (without analyser) ASE emission spectra were acquired in the range extending from 1500 to $1610 \mathrm{~nm}$ and for values of the bias current ranging from $150 \mathrm{~mA}$ to $225 \mathrm{~mA}$.

All the optical elements in this set-up are anti-reflection coated around $1550 \mathrm{~nm}$, and the results discussed in this paper were systematically corrected for the spectral dependency of their transmittance.

\section{RESULTS AND DISCUSSION}

\subsection{Features of the ASE spectra}

Prior to the determination of the Stokes vector $\mathbf{s}$ of ASE, polarisation-resolved measurements of the ASE spectrum for various values of the bias current $I_{\text {bias }}$ were made. Figure 3 thus shows the full ASE spectrum for several values of $I_{\text {bias }}$, as well as its projections along the TE and TM eigenmodes of the structure.

The usual shift of all these spectra towards shorter wavelengths when the bias current increases is observed, in agreement with the expected displacement towards each other of the hole and electron quasi-Fermi levels when the free carrier densities increase. However, an additional feature of those spectra is that the TE and TM spectra exhibit a crossing point at a particular wavelength $\lambda_{c} \simeq 1540 \mathrm{~nm}$. Such a behaviour is characteristic of bulk SOAs presenting tensile strain in their 

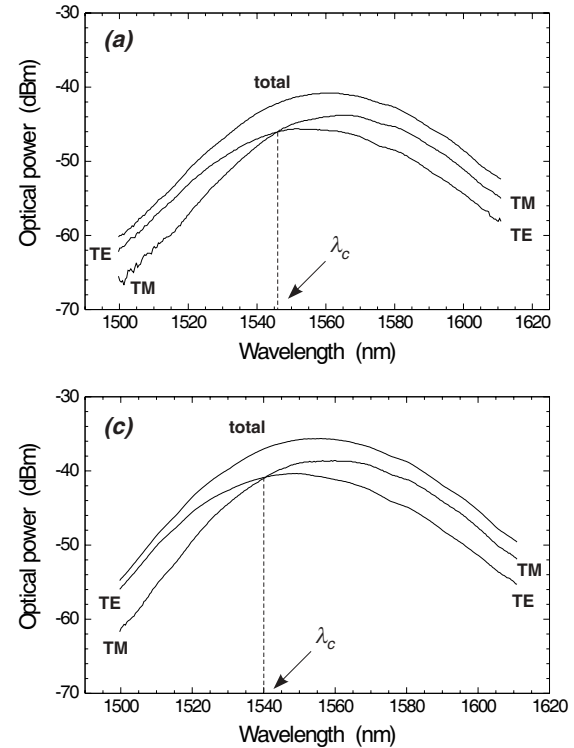
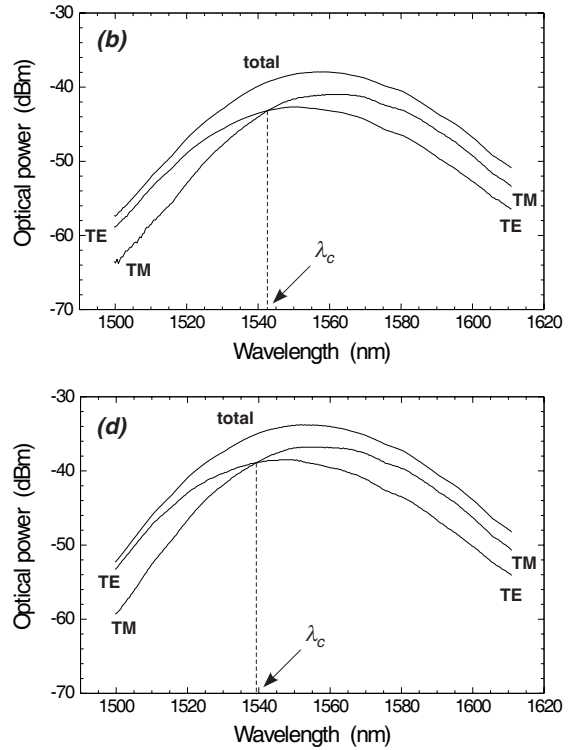

FIG. 3 TE, TM and total optical spectra of the ASE for increasing values of the bias current: $I_{\text {bias }}=150 \mathrm{~mA}$ (a), $175 \mathrm{~mA}$ (b), $200 \mathrm{~mA}$ (c) and $225 \mathrm{~mA}$ (d).

active region, thus a modal anisotropy of the material gain [13]. As both TE and TM spectra simultaneously shift towards shorter wavelengths when $I_{\text {bias }}$ increases, so does their crossing point, as can be verified in Figure 3. This feature will be further discussed in the following.

\subsection{Spectrally-resolved Stokes analysis of the polarimetric behaviour of ASE}

As mentioned above, the full Stokes polarimetric analysis of the ASE emitted by the sample under study consists in determining its Stokes vector $\mathbf{s}$ and its degree of polarisation which will be noted $D O P_{\mathrm{ASE}}$ in the following - using the experimental set-up described in Section 3.

Figure 4 thus shows the spectral variation of $D O P_{\mathrm{ASE}}$, calculated from Eq. (6), for a series of values of the bias current $I_{\text {bias }}$ between 150 and $225 \mathrm{~mA}$. In agreement with the overall shift of the ASE spectra towards shorter wavelengths when $I_{\text {bias }}$ increases, the spectral dependency of its DOP follows the same tendency. As a whole, $D O P_{\mathrm{ASE}}$ assumes larger values as the bias current increases: the more efficient the population inversion, i.e., the larger the density of free carriers pumped into the active region of the SOA, the higher the rate of stimulated electron-hole recombinations leading to the amplified spontaneous generation of photons with a well-defined state of polarisation. This trend is more clearly exhibited in Figure 5, where the average value of $D O P_{\mathrm{ASE}}$ over its whole spectrum is represented as a function of the bias current. A steady, almost linear increase of the averaged value of $D O P_{\mathrm{ASE}}$ with $I_{\text {bias }}$ takes place, showing that ASE evolves from a virtually totally unpolarised source at low pumping levels $\left(D O P_{\mathrm{ASE}} \simeq 0.09\right.$ for $I_{\text {bias }}=100 \mathrm{~mA}$ ) to a signal whose polarised fraction reaches non-negligible values at higher pumping levels, with a DOP up to approximately 0.19 at the maximum experimental bias current of $225 \mathrm{~mA}$.

Furthermore, this spectral dependency exhibits a remarkable

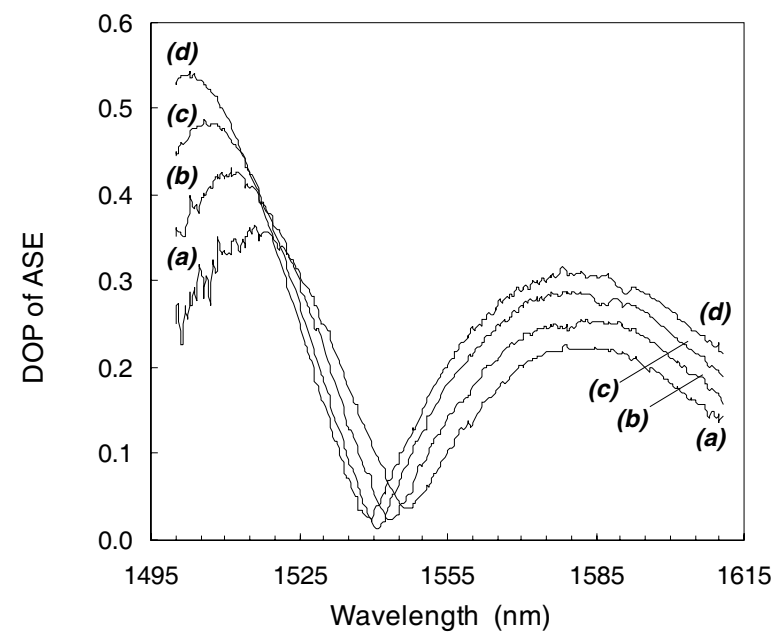

FIG. 4 Spectral dependency of the degree of polarisation (DOP) of ASE for increasing values of the bias current $I_{\text {bias }}=150 \mathrm{~mA}$ (a), $175 \mathrm{~mA}$ (b), $200 \mathrm{~mA}$ (c) and $225 \mathrm{~mA}$ (d).

feature, with $D O P_{\mathrm{ASE}}$ first decreasing towards an almost zero minimum and then increasing again with the wavelength. As can be seen when comparing Figures 3 and 4, the position of this minimum is none other than that of the crossing point $\lambda_{c}$ between the spectral variations of the TE and TM components of ASE. Figure 3 showed a shift of $\lambda_{c}$ towards shorter wavelengths when $I_{\text {bias }}$ increases, and this in turn can be observed with the minimum of $D O P_{\mathrm{ASE}}$. At this particular wavelength, the TE and TM intensity components of ASE are equal and we will see below how this relates to the polarisation state of its polarised fraction. It must be noted that a degree of polarisation equal to zero for ASE in such a structure can arise from two distinct situations: (1) either the signal is made of two orthogonal polarisation states that bear no mutual coherence (i.e., no phase correlation) over the duration of the measurement; (2) or it consists of a large number of pairs of orthogonal polarisation states without any phase correlation - as in nat- 


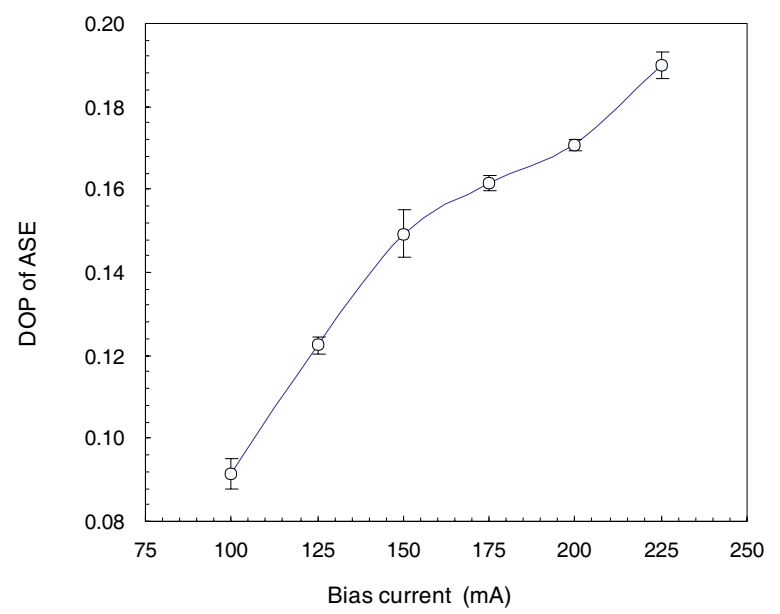

FIG. 5 Degree of polarisation (DOP) of ASE averaged over its whole spectrum as a function of the bias current.

ural light. Given that the structure under study ideally admits two eigenstates, the latter situation seems less likely.

A strong connection exists between the degree of polarisation of ASE and the respective values of the gain coefficient of the structure for its TE and TM eigenmodes. What is usually called the differential gain of the SOA - i.e., the difference between its TE and TM gains - is proportional to the relative difference $|\Delta P| / P_{t}$ (where $\Delta P=P_{T E}-P_{T M}$ ) between the optical powers of the TE and TM components of ASE. That relative difference is, according to Eq. (2), none other than $\left|s_{1}\right| / s_{0}$. Figure 6 compares the behaviours of $D O P_{\mathrm{ASE}}$ and of $\left|s_{1}\right| / s_{0}$ as functions of the wavelength over the whole detection range for a set of bias currents. For each value of $I_{\text {bias }}$ the minimum value of $D O P_{\mathrm{ASE}}$ is reached when the absolute value of $s_{1} / s_{0}$ itself reaches a minimum. The same convergence is observed with the absolute maximum values of $D O P_{\mathrm{ASE}}$ and $s_{1} / s_{0}$. Hence, the higher the differential gain of the structure - i.e., the more one of the eigenmodes dominates the other in terms of optical gain - the more polarised ASE will be. Conversely, when the gain coefficients of the two eigenmodes tend towards similar values, ASE exhibits a low degree of polarisation over the whole spectral range of the detector. We will come back to this convergence in Section 4.3.

Furthermore, looking into the evolution of the polarised fraction of ASE illustrates further the dependence of its polarimetric behaviour upon the gain coefficients of the structure. As described in Section 2, the polarisation state of the polarised fraction of ASE is entirely defined by three parameters, two of which determine the shape (namely the azimuth $\alpha$ and the ellipticity $\epsilon$ ) of its characteristic ellipse. Both parameters can be deduced from the experimental Stokes vector of ASE according to (7) and (8). Figure 7 shows the spectral dependency of these parameters at a bias current of $225 \mathrm{~mA}$. The insets of Figure 7 represent the characteristic ellipse of the polarised fraction of ASE at three particular wavelengths, as deduced from the values of $\alpha$ and $\epsilon$.

One can first observe that the ellipticity of this fraction clearly exhibits the same singularity as $D O P_{\mathrm{ASE}}$ at the crossing-point wavelength $\lambda_{c}$. On the one hand, away from $\lambda_{c}$, the ellipticity angle $\epsilon$ rapidly tends towards a value of almost 0 , which means that the polarised fraction of ASE tends towards a linear state of polarisation, as can also be seen from the insets at $1520 \mathrm{~nm}$ and $1600 \mathrm{~nm}$. On the other hand, the polarisation state of that fraction around $\lambda_{c}$ (i.e., around $1540 \mathrm{~nm}$ for $I_{\text {bias }}=225 \mathrm{~mA}$ ) exhibits a larger ellipticity. A similar behaviour was observed for all the values of the bias current considered in this study.

More importantly, the relationship between the state of polarisation of the polarised fraction of ASE and the gain coefficients of the structure - hence, its ASE emission spectra (as shown in Figure 3) - is even more clearly revealed when observing how the azimuth angle $\alpha$ of the ellipse evolves with the wavelength. For $\lambda<\lambda_{c}$, in the spectral region where the TE component of ASE dominates, the azimuth of its polarised fraction's characteristic ellipse very quickly leans towards 0 , i.e., towards the TE axis of the active zone of the SOA; conversely, when $\lambda>\lambda_{c}$, in the region where the TM component of ASE dominates, $\alpha$ rapidly approaches $\pi / 2$, along the TM axis in the structure - in both cases, even though the dominance of one component of ASE over the other is only gradual (see Figure 3).

The transition from one dominance to the other, around $\lambda_{c}$, is thus rather brutal, and the azimuth assumes the value $\pi / 4$ at that threshold, as is expected from an optical wave whose TE and TM components are equal (see inset at $1540 \mathrm{~nm}$ ). The results depicted in Figure 7 unequivocally show that, on either side of the threshold, the process of amplified spontaneous emission strongly favours one of the eigenstates of the SOA over the other, even when the polarised fraction of ASE is actually small. This spectro-polarimetric behaviour is consistent with the fact that ASE closely depends on the amplification process (hence on the TE and TM gains) within the SOA. Indeed, the photons coherently generated through the amplification of an incoherent spontaneous emission rapidly assume the polarisation state of the dominant mode at a given wavelength. As a result, where the TE (respectively, TM) gain coefficient of the structure dominates, the polarised fraction of ASE tends towards a TE (respectively, TM) polarisation state. It must be noted that the rapidity of the transition from a TEdominated to a TM-dominated ASE does not vary much with the bias current, i.e., with the carrier population available for spontaneous recombinations.

\subsection{Comparison with a standard determination of $D O P_{\text {ASE }}$}

From a practical point of view, it can be noted that a rapid determination of $D O P_{\mathrm{ASE}}$ can be made [14], independently from the full Stokes analysis of its polarised fraction, on the basis of the emission spectrum of the total ASE when compared to those of its TE and TM components. This stems from the fact that, as can readily be seen from the small values of the DOP discussed above, ASE in a SOA is a mostly incoherent signal. In other words, the phase difference $\Delta \phi$ between its TE and TM components is a random, fast-varying function of time at least on the scale of the integration time of any practical detector. As a result, two of the components of its Stokes vectors, 

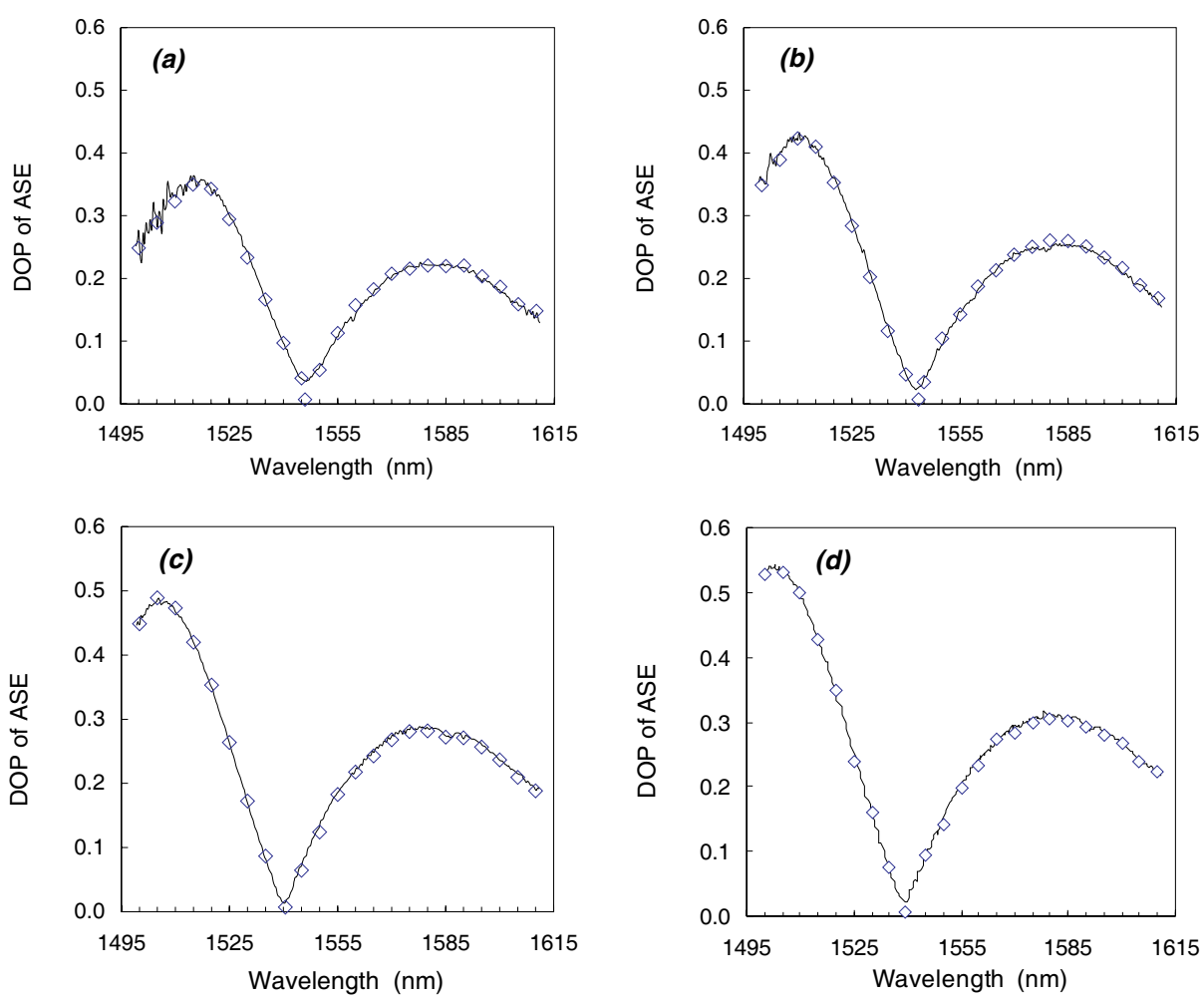

FIG. 6 DOP of ASE obtained from the calculation of $s_{1} / s_{0}$ (diamonds) and from the full set of components of its Stokes vector (solid lines), for increasing values of the bias current: $I_{\text {bias }}=150 \mathrm{~mA}(\mathrm{a}), 175 \mathrm{~mA}$ (b), $200 \mathrm{~mA}$ (c) and $225 \mathrm{~mA}$ (d).

$s_{2}$ and $s_{3}$, are ideally zero, and in practice virtually negligible before the two others [see Eqs. (3) and (4)]. Thus a good approximation of $D O P_{\mathrm{ASE}}$, as defined in (6), can be obtained from the sole values of $s_{0}$ and $s_{1}$, hence from those of $P_{T E}$ and $P_{T M}$, according to:

$$
D O P_{\mathrm{ASE}} \simeq \frac{\sqrt{s_{1}^{2}}}{s_{0}}=\frac{\left|P_{T E}-P_{T M}\right|}{P_{T E}+P_{T M}}=\frac{|\Delta P|}{P_{t}},
$$

where, again, $|\Delta P|$ denotes the difference between the TE and TM fractions of ASE. This result justifies the convergence, observed in the preceding section, between the behaviour of $D O P_{\text {ASE }}$ and that of $s_{1} / s_{0}$.

This is indeed confirmed by the results shown above in Figure 6 for various levels of the bias current, where the exact values of the DOP calculated from the whole Stokes vector of ASE (thin lines, already presented in Figure 4) are confronted to the approximate values deduced from (12) by calculating $\left|s_{1}\right| / s_{0}$ (symbols). All in all, the good agreement between the two sets of results validates the principle of a rapid determination of $D O P_{\mathrm{ASE}}$ solely from the emission spectra of ASE, although it must be clear that only a full Stokes analysis of ASE, as carried out in Section 4.2, can reveal its detailed state of polarisation.

\subsection{Polarimetric behaviour of ASE in the presence of an injected signal}

The preceding results show that the state of polarisation of any polarised fraction of ASE emitted from the structure strongly depends on the dominant gain coefficient of the SOA

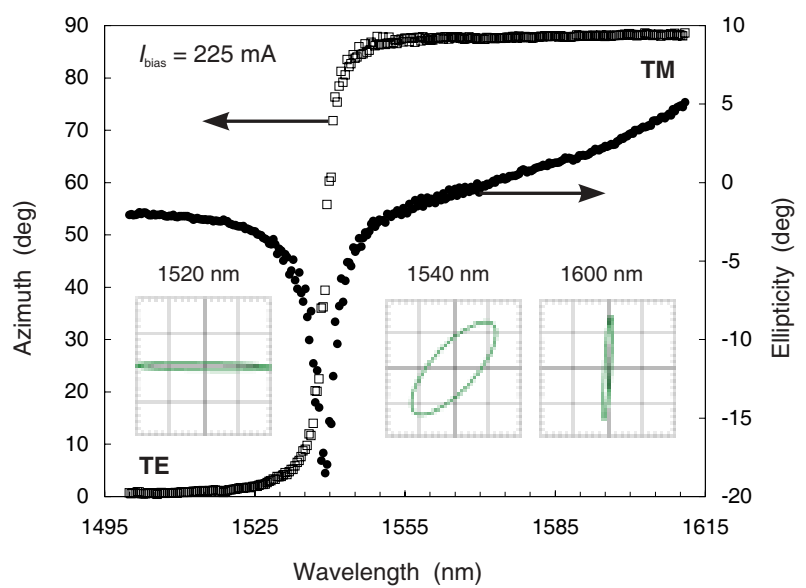

FIG. 7 Spectral dependencies of the polarisation azimuth alpha and ellipticity angle $\epsilon$ of the polarised fraction of ASE for a bias current of $225 \mathrm{~mA}$. The insets show the shape of the characteristic ellipse at three particular wavelengths.

at a given wavelength. A complete study, however, requires to assess the polarimetric behaviour of ASE in presence of an injected optical signal, as is the case in the standard use of a SOA. Furthermore, such an assessment is essential to a description of a SOA within an extended Mueller-Jones formalism that takes ASE into account [11].

In order to address this issue, the Stokes analysis of the ASE emitted from the SOA was performed with an optical signal injected at $\lambda_{i}=1557 \mathrm{~nm}$ (for which the gain of the structure 
is maximum) with either a TE or a TM linear polarisation and for increasing optical powers.

Figure 8 shows the evolution of $D O P_{\mathrm{ASE}}$ spectra for various values of the injected signal power $P_{i}$ between $-30 \mathrm{dBm}$ and $-5 \mathrm{dBm}$ - i.e., between ca. $1 \mu \mathrm{W}$ and $0.3 \mathrm{~mW}$ - for both TE and TM modes. The measurements were made for a bias cur-
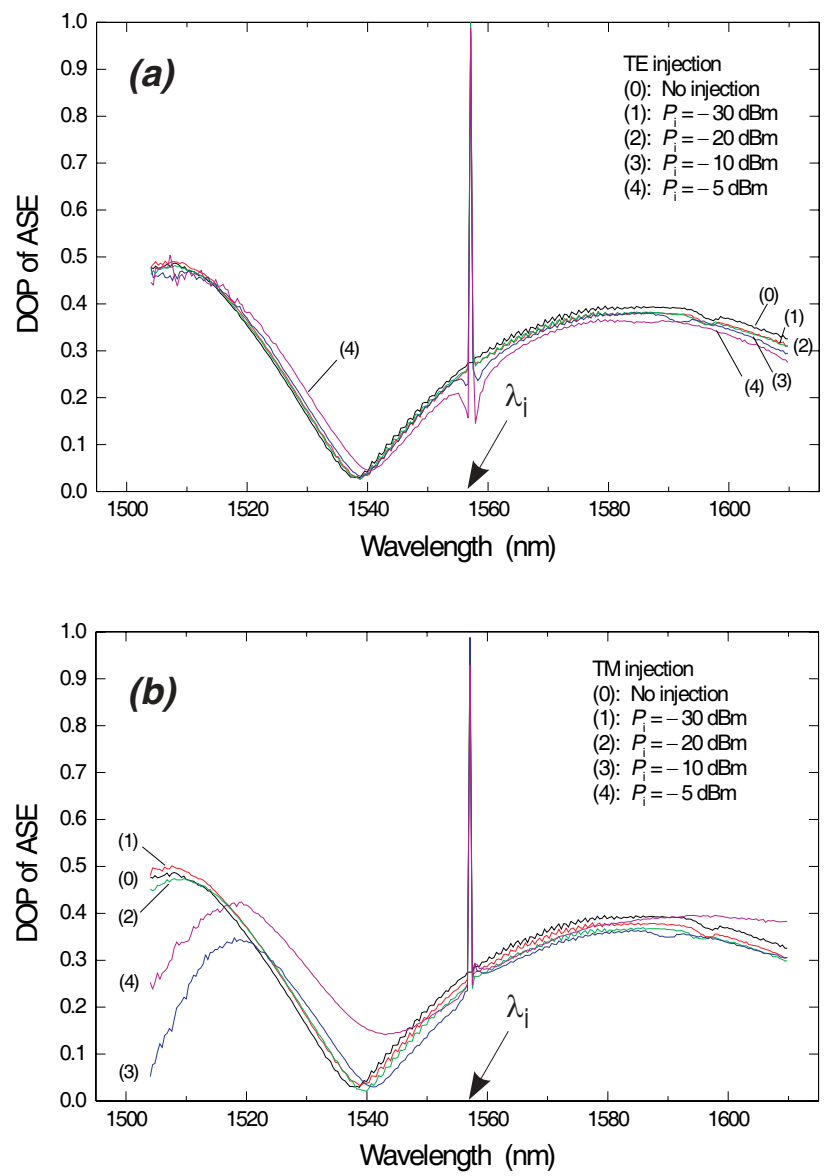

FIG. 8 Spectral dependency of the DOP of ASE in presence of an optical signal injected at $\lambda_{i}$ for various optical powers and with a linear state of polarisation along the TE (a) and TM (b) axis of the SOA.

rent of $225 \mathrm{~mA}$ and were compared to the corresponding TE and TM spectra measured for the same value of $I_{\text {bias }}$ without injected signal (as was shown in Figure 4). As expected, $D O P_{\mathrm{ASE}}$ at $\lambda_{i}$ is essentially equal to the $D O P$ of the injected signal, i.e., equal to 1 , as the fully polarised injected signal largely dominates over ASE. Away from that point, the following features can be observed.

For both polarisation states of the injected signal, $D O P_{\mathrm{ASE}}$ is only slightly affected by the presence of that signal at long wavelengths $\left(\lambda>\lambda_{i}\right)$. Indeed, since amplification and generation of ASE draw from the same carrier reservoirs, carrier populations involved in transitions with an energy lower than $E_{i}$ are little affected by the population depletion provoked by signal injection and its subsequent amplification.

For short wavelengths $\left(\lambda<\lambda_{i}\right)$, however, $D O P_{\mathrm{ASE}}$ spectra obviously experience different variations, depending on the state of polarisation and optical power of the injected signal. These variations are only significant for a TM-polarised in- jected signal, which is consistent with the position of $\lambda_{i}$ on the gain spectra of the structure at $225 \mathrm{~mA}$, i.e., in the region where the TM gain dominates (as can be seen from the ASE spectra shown in Figure 3). In particular, a shift of the $D O P_{\mathrm{ASE}}$ spectrum towards longer wavelengths is observed when the injected optical power increases, which is in agreement with the usual shift of the gain spectra observed in the same conditions. This feature is due to the fact that electrons in the conduction band potentially involved in transitions with an energy higher than $E_{i}$ are likely to thermalize and occupy energy levels liberated by those involved in signal amplification. As a result, spontaneous recombinations occur at lower energy transitions than without injected signal, hence a shift of ASE and $D O P_{\text {ASE }}$ spectra towards longer wavelengths when $P_{i}$ increases. At these short wavelengths, the domination of the TE gain is partly counterbalanced, for higher values of $P_{i}$ and when the injected signal is TM-polarised, by the depletion of electron levels in the conduction band available for spontaneous transitions corresponding to those wavelengths. All in all, this leads to a drop of $D O P_{\mathrm{ASE}}$. In addition, the behaviour of $D O P_{\mathrm{ASE}}$ around the crossing point $\lambda_{c}$ is affected for large values of $P_{i}$ (Figure $8 \mathrm{~b}$ ).

As mentioned above, however, and as can be seen from Figure 8, these effects are much smaller for a TE-polarised injected signal - where the shift in slightly noticeable for $P_{i}=$ $-5 \mathrm{dBm}$ only - than for a TM-polarised injected signal. In the latter case, they are particularly noticeable above $P_{i}=$ $-10 \mathrm{dBm}$ where carrier depletion at $\lambda_{i}$ is important.

Finally, as for the remarkable feature exhibited by $D O P_{\mathrm{ASE}}$ around the crossing point $\lambda_{c}$ and discussed in Section 4.2, i.e., the fact that $D O P_{\mathrm{ASE}}$ nears zero at that point, it mostly remains true with signal injection, except for the highest value of $P_{i}$ for a TM-injected signal, where $D O P_{\text {ASE }}$ departs from zero. This, again, can be attributed to the strongly asymmetric carrier depletion due to signal amplification, i.e., the fact that this phenomenon primarily affects electron-hole pairs likely to be available for TM-polarised ASE generation. This imbalance cancels the compensation observed between TE and TMpolarised ASE observed at $\lambda_{c}$ without injection or even for weak values of $P_{i}$ and thus, according to Eqs. (6) or (12), yields a clearly non-zero value for $D O P_{\mathrm{ASE}}$.

\section{CONCLUSION}

In this paper, the spectro-polarimetric behaviour of ASE in a tensile strained InP/GaInAsP SOA was investigated on the basis of the Stokes formalism. Our experimental results highlight the fact that ASE evolves from an almost totally unpolarised source at low bias current levels (where its degree of polarisation $D O P_{\mathrm{ASE}} \simeq 0.09$ for $I_{\text {bias }}=100 \mathrm{~mA}$ ) to a partly polarised source with a non-negligible polarised fraction for higher values of the bias current $\left(D O P_{\mathrm{ASE}} \simeq 0.19\right.$ for $\left.I_{\text {bias }}=225 \mathrm{~mA}\right)$. Furthermore, these results reveal the spectral dependency of $D O P_{\mathrm{ASE}}$ in a tensile-strained SOA, first decreasing towards an almost zero minimum before increasing again with the wavelength. The position of this minimum corresponds to a crossing point wavelength $\lambda_{c}$ between the TE and TM emission spectra of ASE. This crossing point, due 
to the modal anisotropy of the material gain in the sample, shifts towards shorter wavelengths when the bias current is increased. Moreover, our full Stokes characterization reveals that the characteristic ellipse of the polarised fraction of ASE exhibits the same spectral singularity as the total DOP of ASE, with a brutal transition from a dominant TE mode to a TM one at $\lambda_{c}$. Thus, even when the differential gain of the structure increases gently on either side of $\lambda_{c}$, the process of amplified spontaneous emission strongly favours one of the eigenstates of the SOA over the other. This phenomenon is true even when the polarised fraction of ASE is small. Finally, the same study, performed in presence of an injected signal shows how the dominant mode at the injection wavelength $\lambda_{i}$ modifies the $D O P_{\mathrm{ASE}}$ spectrum for wavelengths below $\lambda_{i}$.

\section{ACKNOWLEDGEMENTS}

The authors wish to thank Profs. J. Cariou and J. Le Bihan for their encouragement and the Council of the region of Brittany, France, for its financial support (PRIR A3CAN4).

\section{References}

[1] M. J. Connelly, Semiconductor Optical Amplifiers (Kluwer Academic Publishers, Dordrecht, 2002).

[2] C. Giuliani, P. Cinguino, and V. Seano, IEEE Photonic. Tech. L. 8, 367-369 (1996).
[3] A. Poustie, Proceedings of the 31st ECOC, 475-478 (2005).

[4] P. Doussière, P. Garabedian, C. Graver, D. Bonnevie, T. Fillion, E. Derouin, M. Monnot, J. G. Provost, D. Leclerc, and M. Klenk, IEEE Photonic. Tech. L. 6, 170-172 (1994).

[5] T. Ito, N. Yoshimoto, K. Magari, and H. Sugiura, IEEE Photonic. Tech. L. 10, 657-659 (1998).

[6] M. Itoh, Y. Shibata, T. Kakitsuka, Y. Kadota, and Y. Tohmori, IEEE Photonic. Tech. L. 14, 765-767 (2004).

[7] M. Itoh, Y. Shibata, T. Kakitsuka, Y. Kadota, H. Sugiura, and Y. Tohmori, J. Lightwave Technol. 24, 1478-1485 (2006).

[8] J. M. Tang, P. S. Spencer, and K. A. Shore, IEEE Photonic. Tech. L. 13, 496-498 (2001)

[9] H. J. S. Dorren, D. Lenstra, Y. Liu, M. T. Hill, and G. D. Khoe, IEEE J. Quantum Elect. 39, 141-147 (2003).

[10] H. Soto, E. Álvarez, C. A. Díaz, J. Topomondzo, D. Erasme, L. Schares, L. Occhi, G. Guekos, and M. Castro, Opt. Commun. 237, 121-131 (2004).

[11] F. F. L. Bentivegna, F. Boulvert, M. Guégan, B. Boulbry, A. Sharaiha, M. Tariaki, F. Pellen, B. Le Jeune, and Y. G. Boucher, SPIE Proc. 5452, 486-497 (2004).

[12] L. F. Tiemeijer, P. J. A. Thijs, T. van Dongen, J. J. M. Binsma, and E. J. Jansen, J. Lightwave Technol. 14, 1524-1533 (1996).

[13] W. Wang, K. Allaart, and D. Lenstra, Electron. Lett. 40, 1602-1603 (2004).

[14] ISO Standard ISO 12005 (2003). 